\title{
Ultra-wideband Tightly Coupled Fractal Octagonal Phased Array Antenna
}

\author{
Eman O. Farhat* Kristian. Z. Adami ${ }^{\dagger} \quad$ Yongwei Zhang ${ }^{\ddagger}$ Anthony K. Brown ${ }^{\S}$ \\ Charles. V. Sammut ף
}

\begin{abstract}
This paper presents a low profile ultrawideband tightly coupled phased array antenna with integrated feedlines. The aperture array consists of planar element pairs with fractal geometry. In each element these pairs are set orthogonal to each other for dual polarisation. The design is an array of closely capacitively coupled pairs of fractal octagonal rings. The adjustment of the capacitive load at the tip end of the elements and the strong mutual coupling between the elements, enables a wideband conformal performance. Adding a ground plane below the array partly compensates for the frequency variation of the array impedance, providing further enhancement in the array bandwidth. Additional improvement is achieved by placing another layer of conductive elements at a defined distance above the radiating elements. A Genetic Algorithm was scripted in MATLAB and combined with the HFSS simulator, providing an easy optimisation tool across the operational bandwidth for the array unit cell design parameters. The proposed antenna shows a wide-scanning ability with a low cross-polarisation level over a wide bandwidth.
\end{abstract}

\section{INTRODUCTION}

Recently, low-profile conformal array designs are attracting much attention for the development of Ultra Wideband (UWB) phased arrays capable of supporting communications, radar functions, warfare, and radio astronomy applications such as the square kilometer array (SKA) [1]. However, designing planar low-cost phased array apertures that is concurrently broadband and low-profile with low cross polarisation has been a continuing challenge.

The theoretical approach of broadband antennas was presented by Wheeler in [2], The Current Sheet Array (CSA) concept utilizes the mutual coupling between the adjacent radiating elements array to enable wideband conformal performance. Munk et $a l .[3]$,[4],emulated CSA concept, by designing a pe-

\footnotetext{
*Department of Physics, University of Malta, Msida Malta, e-mail: efar0026@um.edu.mt

${ }^{\dagger}$ Department of Astrophysics, University of Oxford, Oxford, e-mail: kza@astro.ox.ac.uk

${ }^{\ddagger}$ School of Electrical and Electronic Engineering, The University of Manchester, Manchester, M13 9PL, U. K., email: david.zhang@manchester.ac.uk

$\S$ School of Electrical and Electronic Engineering, The University of Manchester, Manchester, M13 9PL, U. K., email: anthony.brown@manchester.ac.uk

IDepartment of Physics, University of Malta, Msida Malta, e-mail: charles.v.sammut@um.edu.mt
}

riodic planar array of closely packed dipoles placed at a distance of $<\frac{\lambda}{4}$ (at the centre of the operating frequency band) above an infinite ground plane. Munk's array has demonstrated large bandwidths about 3 : 1 with VSWR $<2$. The neighboring dipoles are designed using large capacitors and are fed at the centre via feed organizer. However, large external baluns are required.

Other well-known UWB phased array antennas such as Tem horn[5], bunny-ear[6], Vivaldi[7]feature a third dimension which plays a role in their broadband performance. However, these elements are costly and also difficult to fabricate. On the other hand, ORA [8] consists of closely spaced octagonal ring elements, exhibits a ratio of $3.3: 1$ with VSWR $<2$ bandwidth.

The paper is orgonaised as follows:In Section 2, the concept of the array is presented. Section 3 describes the new antenna design with integrated feeding lines. The analysis of the infinite array is presented in Section 4, with the conclusions described in Section 5 .

\section{UWB tightly coupled phased array con- cept}

A novel class of antennas referred to as Tightly Coupled Phased Arrays (TCPAs), are practical implementations of the CSA concept. They exhibit well-behaved impedance and radiation patterns over a wide bandwidth. The basic idea of TCPAs is bunching very small elements very close to each other, utilizing the strong capacitive mutual coupling between them to counteract the ground plane inductance. This counteraction occurs at a single frequency, yielding a doubling of bandwidth performance. However, the array is limited at higher frequencies by the presence of grating lobes as well as the $0.5 \lambda_{H}$ reflection from the ground plane where $\lambda_{H}$ is the wavelength at the highest operational frequency. Additionally, the use of orthogonal excitation ports allow for arbitrary vertical and horizontal polarisation. Furthermore, feeding groups of array elements allows for beam steering capabilities.

Forming broadband arrays from TCPAs has been 


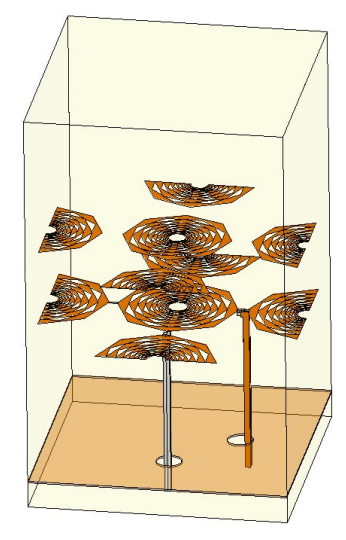

Figure 1: Geometry of the unit cell with the feeding lines.

extended to various types of electrically small elements such as bowties and proven to be applicable for spirals[9] , pixelated elements [10], and tapered slots[11]. Munk initiated this class of TCPAs with his idea of capacitively-coupled dipoles array embedded in dielectric layers above the ground plane, and was implemented to practice at Harris [12]. For further increasing the CSA array bandwidth, Munk has suggested several ways. Among them were the use of dielectric superstrates, magnetic substrates, or lossy absorbers layers placed on the ground plane. However, using low-loss dielectric superstrates enhances the CSA bandwidth at the expense of significantly increasing its height.

\section{Antenna design}

The self-similarity of fractals is the cause of multiband and broadband properties. Therefore, our proposed elements are based on a geometrical fractal, a form of iterative octagon rings subdivided in parts such each part is a reduced size copy of the whole. The aperture array consists of periodically closely spaced elements, which are octagonal rings with a fractal configuration backed at a defined distance by a ground plane. The strongly capacitive coupling between adjacent rings is implemented by inserting bulk capacitors between the tip end portions of the elements. To improve the impedance matching of the individual antenna elements over a wide band frequency range, an impedance matching layer comprised of scaled down periodic conductive rings is designed and placed over the radiating aperture. Figures 1 illustrates the proposed array unit cell structure in HFSS. The rings are linearly positioned across each other sharing the centre ring. Dual polarisation is realized by using

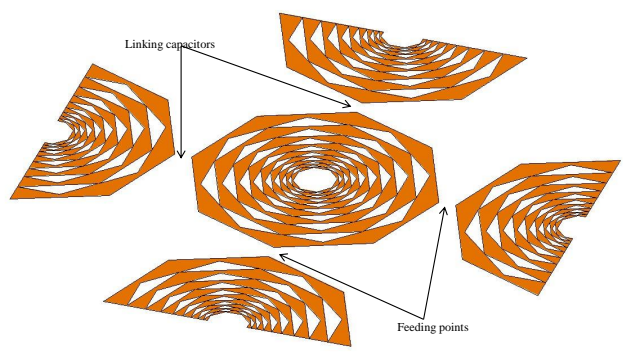

Figure 2: The unit cell radiating element, illustrating feeding points and also linking capacitors position.

two-orthogonal pair of rings, as shown in Figures 2. Arrays with a significant inter-element coupling are usually accompanied by grating lobes, which causes a reduction in gain or impedance discontinuity. In order to avoid grating lobes the inter-element spacing is set $<\frac{\lambda}{2}$ at the highest frequency. Applying this with an array lattice of one-half wavelength allows the main beam to be scanned over all space without grating lobes.

In order to avoid boresight radiation cancellation from the ground plane image current which occurs when the ground plane separation is $0.5 \lambda_{H}$, the ground plane distance should be $<0.4 \lambda_{H}$. Since the array elements form a balanced structure a new stripline structure introduced in [8] is been used to feed the array at each element as depicted in Figures 3 . The feeding line is inserted prior to the end edge of a pair of ring radiators at the top part of the feeding line while the inner conductor of the stripline is soldered to the other pair ring (centre ring). The input impedance of the candidate antenna is about $120 \Omega$. Therefore, an impedance transformation circuit is designed in the stripline to allow the $120 \Omega$ balanced feed to radiating elements to be transformed to a single end $50 \Omega$ system. This feeding scheme is a single-ended structure which can effectively avoid the common-mode effect, significant low insertion loss, ease for LNA integration, and a convenient solution for large scale realization. Additionally, no baluns are required to feed the antenna.

\section{Infinite array simulation performance}

The corresponding array is designed to operate over frequency bandwidth ranging from $250 \mathrm{M} \mathrm{Hz}-$ $1.25 \mathrm{GHz}$. The unit cell is $0.5 \lambda_{H}(160 \mathrm{~mm})$ element 


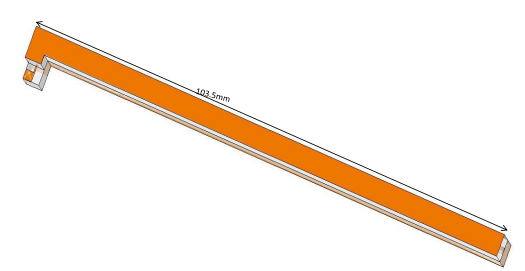

Figure 3: The stripline configuration to feed the array elements.

spacing (at 1.1GHz) and the radiating elements are placed $0.32 \lambda_{H}(86 \mathrm{~mm})$ above a PEC ground plane. Capacitively mutual coupling is used to cancel the inductive ground plane. Low frequency bandwidth performance is improved by inserting a bulk capacitor of $0.8 p F$ between the tips of the rings as shown in Figures 2 (5mm gap between the rings). The matching layer is then merely a scaled down version of the radiator layer, and the distance from this layer to the radiating layer is $(46 \mathrm{~mm})$. A commercial full wave solver, HFSS v12, with periodic boundary conditions is used to simulate an infinite array. The previous mentioned parameters are optimum parameters, obtained through a Genetic Algorithm (GA) optimization procedure scripted in Matlab. The objective of this optimization is to reduce the S11 parameter (reflection coefficient) values over the operating frequency band of our candidate antenna array design. Matlab controls HFSS, designing physical dimensions of the array unit cell generated by the GA and then returns S11 parameters to Matlab. This optimisation method provides an easy tool and a convenient way ofoptimising the infinite array unit cell for optimal design parameters.

To further demonstrate the validity of the capacitively-coupled Fractal Octagonal Rings phased Array (FOAR) antenna, a full-wave simulation data is presented. The element geometry was initially designed for optimal performance within an infinite array. The same unit cell configuration is simulated in both CST Microwave Studio and HFSS. Figures 4 shows good agreement in the reflection coefficient performance for an element in a dual-polarised infinite array between the CST and HFSS simulations. The scan performance of the array is shown in Figures 5, showing that the maximum scan volume for the array is $\pm 45^{\circ}$ in all directions. A stable cross polarisation performance is illustrated in Figures 6, scanned in four directions. The surface current analysis shown in Figures 7 reveals that the current flows along the edges of the fractal patches. Furthermore, it is noted from Figures 8 that the loss in gain is low from broadside to $45^{\circ}$ is about $2.5 d B$ on average over the frequency band.

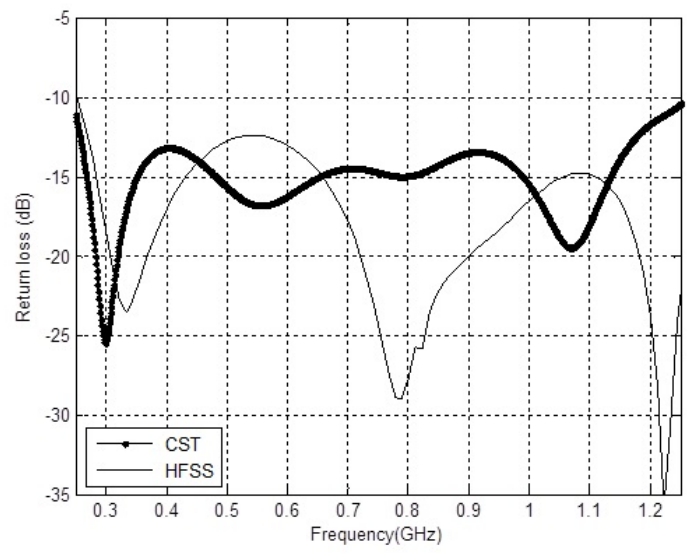

Figure 4: The broadside scan performance reflection coefficient for an element in the infinite array simulated in both CST and HSFF.

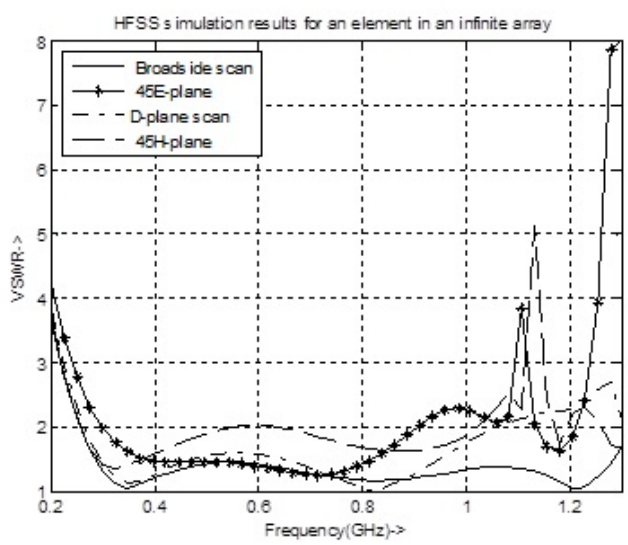

Figure 5: The scan performance of the active VSWR parameters for an element in the infinite array.

\section{Conclusion}

A simple planar configuration with a fractal geometry for a broadband aperture has been presented. The proposed elements array have shown to improve tightly coupled array bandwidth up to $5: 1$. The array demonstrated a stable low level crosspolarisation (below $15 \mathrm{~dB}$ ) within a $\pm 45^{\circ}$ scan volume range, and low loss of gain. This antenna array 


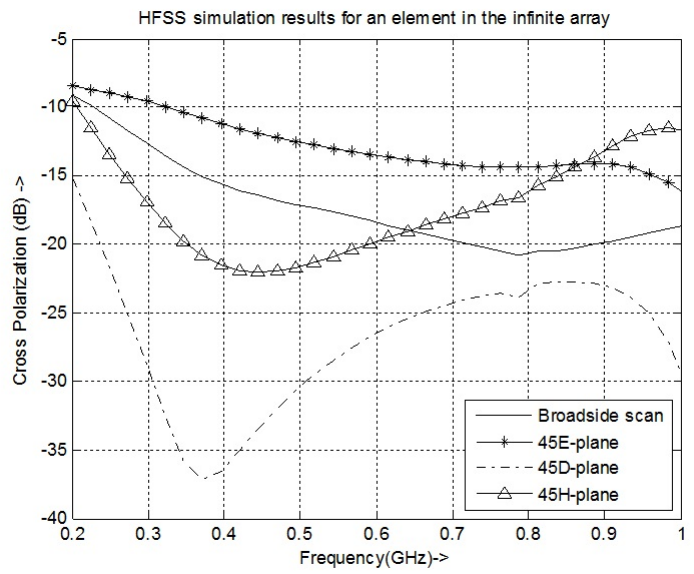

Figure 6: The cross polarization level in $\mathrm{dB}$ for an element in the infinite dual polarized array.
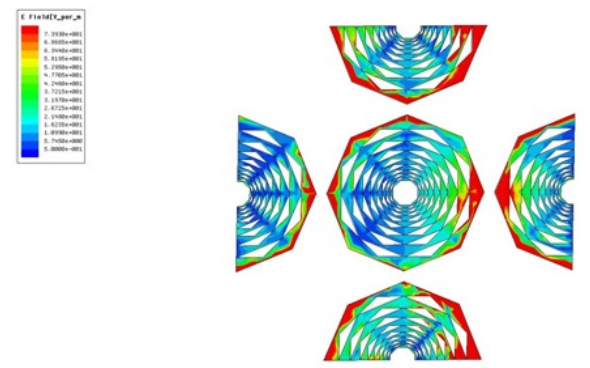

Figure 7: The surface current at $1.28 \mathrm{GHz}$ for broadside scan of an element in the infinite array.

can be easily fabricated in two sheet layers of conductive fractal rings separated by foam. Since it is inherently low-profile we expect the array to be constructed at low cost and hence it is extensible for large scale arrays.

\section{Acknowledgments}

The authors would like to thank Yongwei Zhang and Anthony K. Brown for their kind cooperation.

\section{References}

[1] P. E. Dewdney, P. J. Hall, R. T. Schilizzi, T. Joseph, and L. W. Lazio, "The square kilometre array," Proc.IEEE, vol. 97, no. 8, pp. 1482-1496, 2009.

[2] H. A. Wheeler, "Simple relations derived from a phased-array antenna made of an infinite current sheet," Antennas and Propagation, IEEE Transaction on, vol. 13, no. 4, pp. 506514, 1965.

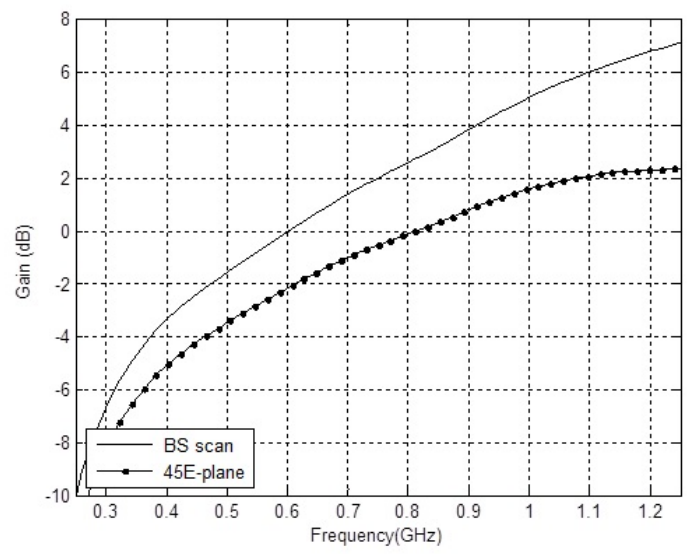

Figure 8: The gain for the immersed elements in an infinite array.

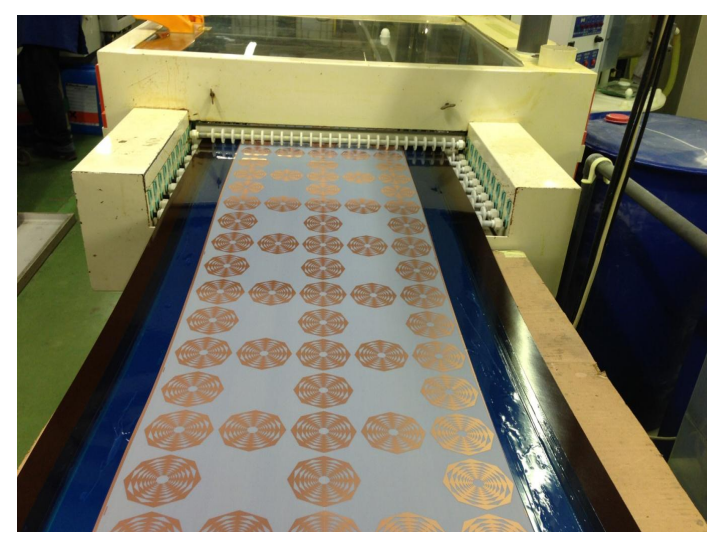

Figure 9: Manufacturing of the prototype of $12 \times 12$ element finite array.

[3] B. A. Munk, Finite Antenna Array and Fss. New York: John Wiley, 2003.

[4] B. Munk, R. Taylor, T. Durharn, W. Croswell, B. Pigon, R. Boozer, S. Brown, M. Jones, J. Pryor, S. Ortiz, J. Rawnick, K. Krebs, M. Vanstrum, G. Gothard, and D. Wiebelt, "A low-profile broadband phased array antenna," in Antennas and Propagation Society International Symposium, 2003. IEEE, vol. 2, june 2003, pp. 448 - 451 vol.2.

[5] E. Holzman, "A wide band tem horn array radiator with a novel microstrip feed," in Phased Array Systems and Technology, 2000. Proceedings. 2000 IEEE International Conference on, 2000 , pp. $441-444$.

[6] J. Lee, S. Livingston, and R. Koenig, "A lowprofile wide-band (5:1) dual-pol array," Antennas and Wireless Propagation Letters, IEEE, vol. 2, no. 1, pp. $46-49,2003$.

[7] J. Shin and D. Schaubert, "A parameter study 
of stripline-fed vivaldi notch-antenna arrays," Antennas and Propagation, IEEE Transactions on, vol. 47 , no. 5 , pp. $879-886$, may 1999.

[8] Y. Zhang and A. Brown, "Octagonal ring antenna for a compact dual-polarized aperture array," Antennas and Propagation, IEEE Transactions on, vol. 59, no. 10, pp. 3927 3932 , oct. 2011.

[9] Riddle, et al., "Conformal, low rcs, wideband, phased array antenna for satelite communications applications," US Patent 6,300,918, 9 Octobrer 2001.

[10] Maloney, et al., "Fragmented aperture antennas and broadband antenna ground planes," US Patent 6,323,809, 27 November 2001.

[11] Kragalott, et al., "Design of a 5:1 bandwidth stripline notch array from fdtd analysis," IEEE Trans. Antennas Propagat., vol. Ap-48, no. 11, pp. 1733-1741, November 2000.

[12] Taylor, et al., "Wideband phased array antenna and associated methods," US Patent 6,512,486, 28 January 2003. 